\title{
Bioanalysis
}

\section{White Paper on recent issues in bioanalysis: a full immersion in bioanalysis (Part 3 - LBA and immunogenicity)}

\begin{abstract}
The 20148th Workshop on Recent Issues in Bioanalysis (8th WRIB), a 5-day full immersion in the evolving field of bioanalysis, took place in Universal City, California, USA. Close to 500 professionals from pharmaceutical and biopharmaceutical companies, contract research organizations and regulatory agencies worldwide convened to share, review, discuss and agree on approaches to address current issues of interest in bioanalysis. The topics covered included both small and large molecules, and involved LCMS, hybrid LBA/LCMS, LBA approaches and immunogenicity. From the prolific discussions held during the workshop, specific recommendations are presented in this 2014 White Paper. As with the previous years' editions, this paper acts as a practical tool to help the bioanalytical community continue advances in scientific excellence, improved quality and better regulatory compliance. Due to its length, the 2014 edition of this comprehensive White Paper has been divided into three parts for editorial reasons. This publication (Part 3 ) covers the recommendations for Large molecules bioanalysis using LBA and Immunogenicity. Part 1 (Small molecules bioanalysis using LCMS) and Part 2 (Hybrid LBA/LCMS, Electronic Laboratory Notebook and Regulatory Agencies' Input) were published in the Bioanalysis issues 6(22) and 6(23), respectively.
\end{abstract}

The 8th Workshop on Recent Issues in Bioanalysis (WRIB) was hosted in Universal City, California, USA, on 10-14 March, 2014. The workshop included three sequential core workshop days and six training courses which together spanned an entire week in order to allow exhaustive and thorough coverage of all major issues in bioanalysis. This gathering brought together close to 500 professionals, representing over 200 companies, to share and discuss current topics of interest in the field of bioanalysis. Attendance included a wide diversity of industry experts from pharmaceutical and biopharmaceutical companies, contract research organizations (CROs) and multiple international regulatory agencies.

The actively contributing chairs in the 2014 edition of the WRIB were Eric Fluhler (Pfizer, USA), Olivier Le Blaye (ANSM, France), Dawn Dufield (Pfizer, USA), Lakshmi Amaravadi (Biogen Idec, USA), Lauren Stevenson (Biogen Idec, USA) and Fabio Garofolo (Algorithme Pharma, Canada).
The numerous regulatory agency representatives who contributed to the 8th WRIB included Sam Haidar (US FDA), Amy Rosenberg (US FDA), Susan Kirshner (US FDA), Laura Salazar-Fontana (US FDA), Mark Bustard (Health Canada), Jan Welink (Dutch Medi-cines Evaluation Board [MEB] and European Medicines Agency [EMA]), Olivier Le Blaye (French National Agency for Medicines and Health Products Safety [ANSM], France), Ronald Bauer (Agency for Health and Food Safety [AGES], Austria), Katalina Mettke (Federal Institute for Drugs and Medical Devices [BfArM], Germany), Emma Whale (Medicines and Healthcare products Regulatory Agency [MHRA], UK), Jason Wakelin-Smith (MHRA, UK), Noriko Katori (Ministry of Health, Labour, and Welfare - National Institute of Health Sciences [MHLW-NIHS], Japan), and Akiko Ishii-Watabe (MHLW-NIHS, Japan).

As with prior WRIB editions [1-6], a substantial number of topics were addressed during the workshop and distilled into a series of relevant recommendations. In the present
Lauren Stevenson', Lakshmi Amaravadi', Heather Myler², Laura Salazar-Fontana ${ }^{\ddagger, 3}$, Boris Gorovits ${ }^{4}$, Susan Kirshner ${ }^{\S, 3}$, Li Xue ${ }^{4}$, Fabio Garofolo*,5, Stephen C Alley ${ }^{6}$, Theingi Thway ${ }^{7}$, Alison Joyce ${ }^{4}$, Surendra Bansal ${ }^{8}$, Chris Beaver ${ }^{9}$, Annik Bergeron ${ }^{5}$, Xiao-Yan Cai ${ }^{10}$, Laura Cojocaru"1, Binodh DeSilva ${ }^{2}$, Isabelle Dumont ${ }^{5}$, Eric Fluhler ${ }^{12}$, Stephanie Fraser $^{13}$, Dominique Gouty $^{14}$, Swati Gupta' ${ }^{15}$, Sam Haidar $^{3}$, Roger Hayes ${ }^{16}$, Benno Ingelse ${ }^{17}$, Akiko Ishii-Watabe ${ }^{18}$, Surinder Kaur $^{19}$, Lindsay King ${ }^{13}$, Omar Laterza $^{20}$, Sheldon Leung ${ }^{4}$, Ann Lévesque ${ }^{21}$, Mark $\mathrm{Ma}^{7}$, Corinne Petit-Frere ${ }^{22}$, Renuka Pillutla ${ }^{2}$, Mark Rose ${ }^{7}$, Gary Schultz ${ }^{23}$, John Smeraglia ${ }^{24}$, Steven Swanson', Albert Torri ${ }^{25}$, Faye Vazvaei ${ }^{8}$, Jason Wakelin-Smith ${ }^{26}$, Amanda Wilson' ${ }^{27}$, Eric Woolf ${ }^{28}$ \& Tong-Yuan Yang 29 *Author for correspondence: Tel.: +1 4509736077 Fax: +1 4509732446 fgarofolo@algopharm.com Author affiliations can be found at the end of this article

The views expressed in this article are those of the authors and do not reflect official policy of the US FDA, Europe EMA, Health Canada, France ANSM, The Netherlands MEB, Germany BfArM, Austria AGES, Japan MHWL and UK MHRA. No official endorsement by the FDA, EMA, Health Canada, ANSM, MEB, BfArM, AGES, MHWL or MHRA is intended or should be inferred.

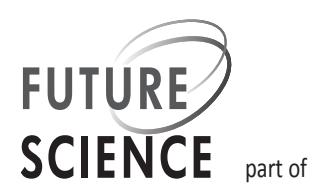




\begin{tabular}{|c|c|}
\hline \multicolumn{2}{|l|}{ Acronyms } \\
\hline Abbreviation & Definition \\
\hline$A D C$ & Antibody-drug conjugate \\
\hline BMV & Bioanalytical method validation \\
\hline CDR & $\begin{array}{l}\text { Complementarity determining } \\
\text { region }\end{array}$ \\
\hline DAR & Drug to Antibody Ratio \\
\hline ELISA & $\begin{array}{l}\text { Enzyme-linked immunosorbent } \\
\text { assay }\end{array}$ \\
\hline ISR & Incurred sample reanalysis \\
\hline LBA & Ligand binding assay \\
\hline LCMS & $\begin{array}{l}\text { Liquid chromatography mass } \\
\text { spectrometry }\end{array}$ \\
\hline LLOQ & Lower limit of quantification \\
\hline mAb & Monoclonal antibody \\
\hline MD & Method development \\
\hline MDB & multi-domain biotherapeutic \\
\hline MOA & Mechanism of action \\
\hline MRD & Minimum required dilution \\
\hline NAb & Neutralizing antibody \\
\hline PEG & Polyethylene glycol \\
\hline Pre-Ab & Pre-existing Antibodies \\
\hline PD & Pharmacodynamic \\
\hline PK & Pharmacokinetic \\
\hline QC & Quality control samples \\
\hline SPE & Solid phase extraction \\
\hline WRIB & $\begin{array}{l}\text { Workshop on Recent Issues in } \\
\text { Bioanalysis }\end{array}$ \\
\hline
\end{tabular}

White Paper, the exchanges, consensus and resulting recommendations on 36 recent issues ('hot' topics) in bioanalysis are presented. These 36 topics are distributed within the following areas:

- Small molecules by LCMS:

- Emerging technologies (three topics);

$=$ Bioanalytical challenges (height topics).

- Hybrid LBA/LCMS:

= Large molecules by LCMS (five topics);

$=$ Antibody-drug conjugates (three topics);

= Protein biomarkers by LCMS (five topics).

- Large molecules by LBA:

- Immunogenicity (five topics);
= PK LBA bioanalytical challenges (six topics).

- Electronic Laboratory Notebooks (one topic).

Following the recommendations on the above topics, an additional section of this White Paper focuses specifically on several key inputs from regulatory agencies.

Due to its length, the 2014 edition of this comprehensive White Paper has been divided into three parts for editorial reasons. This publication (Part 3) covers the recommendations for Large molecules bioanalysis using LBA and Immunogenicity. Part 1 (Small molecules bioanalysis using LCMS) and Part 2 (Hybrid LBA/LCMS, Electronic Laboratory Notebook and Regulatory Agencies' Input) were published in the Bioanalysis issues 6(22) and 6(23), respectively.

\section{Large molecules by LBA discussion topics}

\section{Immunogenicity}

Characterization of immunogenicity responses towards antibody-drug conjugates (ADCs)

Do immunogenicity assays need to be specific for each species (different payload levels)? What is the industry experience with anti-drug antibody (ADA) domain specificity evaluation? Do regulatory agencies expect domain specificity evaluation? Should the industry conduct domain specificity evaluation going forward?

\section{Characterization of Pre-existing Antibody (Pre-Ab) responses}

What data are available concerning the specificity of naturally existing antibodies that cross-react with therapeutic proteins and treatment-emergent ADA? What correlative data are available to link IgG-mediated hypersensitivity reactions to treatment-emergent ADA? When is determination of ADA isotype and domain specificity testing merited? What strategies are used to manage Pre-Abs in preclinical and clinical phases?

Challenges with bioanalysis of anti-polyethylene glycol (PEG) antibodies and ADA specific to PEG What are the challenges encountered when developing assays to detect anti-PEG antibodies (i.e. crossreactivity of detergents used in assay buffers, matrix interference including human IgG or PEG analogs)? What is the incidence of anti-PEG antibodies among human populations? Does it vary with age, condition and ethnicity? What is the predominant isotype of the anti-PEG antibodies reported in the clinic and in the literature? What mitigation strategies could be used to reduce their potential impact in safety/efficacy? What type of mitigation strategies should be in place when 
anti-PEG antibodies are detected in a large number of drug-naïve patients?

\section{Neutralizing Antibody (NAb) assays}

When is it important to have an assay that identifies NAbs? Under what circumstances is a cell-based assay warranted rather than an LBA? What functional endpoints make reasonable NAb assays? What are the most important characteristics for a strong NAb assay?

Impact of circulating drug on ADA measurement What are the industry standards on drug tolerance, its impact on interpretation of ADA results, and mitigation strategies? What are the new approaches to speed up early preclinical immunogenicity assessments?

\section{PK LBA bioanalytical challenges}

\section{Bioanalysis of multifunctional biotherapeutics} and biotransformation of biotherapeutics

What are the proper methods to fully characterize the PK of multifunctional domain molecules? What is the fit-for-purpose immunogenicity strategy that should be implemented for this type of molecule? How to evaluate the long-term stability of this type of molecule in vitro and in vivo? With regards to biotransformation of biotherapeutics, what needs to be measured and why? How to distinguish between charge variants? Does it depend on PK/PD exposure-response relationship? What is the impact of biotransformation (safety, efficacy, stability/half-life)?

\section{Challenges with bioanalysis of PEGylatedproteins by LBA}

What are the assay development and validation considerations for PK assays when there are limited options for specific reagents against PEG molecules? What is the industry standard? What is the effect of PEG on free and total forms?

\section{Transition from early to later PK assays}

Are there challenges in the transition from early to later assays? What is the current industry practice when changing from valency insensitive assays to assays that measure a dominant/active species? How to manage unexpected PK profiles related to changes in assay format?

\section{Overcoming interferences in PK LBA}

What are the current practices for initially evaluating LBA interference during assay development and then mitigating these interferences? Are free or bound assays really necessary, especially for preclinical studies? What are the pros and cons of LCMS versus LBA?

\section{Key terms}

Immunogenicity: The ability of a particular biotherapeutic substance to provoke an immune response, either by humoral production of anti-drug antibodies or through cellular-based immune responses.

Payload: In antibody-drug conjugates, the payload represents the biologically active cytotoxic drug that is conjugated with the antibody.

PEGylated proteins: Proteins modified through covalent attachment of polyethylene glycol polymer chains to improve their pharmacological properties, such as improved drug solubility, increased drug stability, extended circulating life and enhanced protection from proteolytic degradation to achieve reduced dosage frequency without diminished efficacy, and potentially reduced toxicity.

Specifically, do interference issues with LBA justify switching to LCMS methods?

\section{PK assay and immunogenicity}

What is the impact of ADA on measuring serum drug levels? Is it possible to have a complete dissociation of the antibody/drug complex using a pre-treatment? Will it allow interpretation of an accurate concentration? How are data from free assays being handled, knowing that the equilibrium may be disturbed with dilution? Should ADA interference in PK assays routinely be examined? Do strategies need to be employed to mitigate ADA interference? Should ADA tolerance for PK LBAs be examined/reported?

\section{Follow-up on LBA part of the 2013 draft FDA Guidance on bioanalytical method validation (BMV)}

How should the nominal concentrations of protein drugs be determined? Is it useful to include additional quality control (QC) samples when study sample concentrations are clustered in a narrow range of the standard curve? For sample analysis using LBA, are we ready to use a single replicate for sample analysis?

\section{Large molecules by LBA discussions, consensus \& conclusions}

Immunogenicity

\section{Characterization of immunogenicity responses toward ADCs}

ADCs belong to the second generation of biotherapeutic compounds characterized by complex multidomain structure and multistep mode of action. Other examples of multi-domain biotherapeutics (MDBs) are bi-specific antibodies, PEGylated proteins/peptides and fusion proteins. $\mathrm{MDBs}$, including $\mathrm{ADCs}$, are capable of inducing an immune response similar to 
any other type of biotherapeutic. Antibody response to ADCs should be carefully evaluated non-clinically and in human studies. A recent publication [7] addressed potential MDB-related risk factors and approaches to be considered when evaluating and characterizing antibody responses to MDBs. Potential compound-related immunogenicity risk factors that are specific for ADCs based on their multi-domain nature are as follows:

- Novel linear and structural epitopes, for example non-human amino acid sequences found on the tether or the linker, inter-domain and linkerdomain interface structures and surfaces. Such novel non-human epitopes have an elevated antigenic potential;

- Small molecule toxin attachment to an antibody molecule may result in increased half-life of the toxin and therefore has the potential to increase the incidence or magnitude of an immunogenic response through the improved presentation of small molecule antigen to antigen-presenting cells (hapten effect) [7,8];

- Formation of large immune complexes based on the multivalent nature of antibodies and availability of multiple epitopes on the ADC compound. Large circulating immune complexes may potentially form and lead to risk of immune complex toxicity;

- Plants or bacterially derived toxins can have significant immunogenicity potential.

As ADC compounds are commonly developed for oncology indications, a relatively low patient immune competence should be considered for the overall immunogenicity risk assessment.

During anti-ADC antibody testing, a tier-based approach should be applied, which minimally includes screening and confirmatory tests [9]. Anti-ADC assays with sufficient sensitivity, selectivity and drug tolerance are typically expected. Neutralizing activity of anti-ADC antibody should be considered specifically in the clinical phase of development. Additional characterization of domain specificity of anti-ADC antibody should be considered as dictated by the immunogenicity risk-based analysis for the specific compound. Specificity of the anti-ADC responses to the $m A b$, the small molecule drug or the drug/linker domains can be determined. Although ADCs are expected to be a heterogeneous mixture of components with variable drug loading, a separate testing of immunogenicity potential for various drug to antibody ratio (DAR) components is not proposed.

Various domain specificity tests have been described including competitive inhibition and separate domain specific screening tests $[10,11]$. Typically, availability of a positive control (PC) specifically developed for the respective domain specificity test is expected. Competitive inhibition-based domain characterization assays are typically set up as a confirmatory test where an excess of the domain in question is spiked into the sample. A reduction in the assay signal indicates domain specificity of the anti-ADC antibody. As with any typical confirmatory assay, domain specificity tests require a confirmatory cut-point to be set up during assay development or validation phase [9]. Since the antiADC response is polyclonal, a mixed type of specificity should be expected and has been reported [10].

Antibody domain specificity testing enables a better understanding of the nature of the anti-ADC immune response including the identification of critical contributing factors. This understanding could also enable the ability to design improved testing strategies during later compound development phases and could provide valuable information to be applied during compound re-engineering. This testing might also improve the prediction of potential clinical sequelae.

For example, anti-ado-trastuzumab emtansine (T-DM1) antibodies were evaluated in a tiered-based approach [12] with approximately 5\% of the patients testing positive. Domain specificity characterization tests showed that majority of the anti-T-DM1 antibodies were directed to epitopes not found on the antibody component of the ADC, but were directed toward the linker-drug or conformational alterations introduced at the point of conjugation. Importantly, there was no significant impact of the anti-T-DM1 antibodies on safety, pharmacokinetics or efficacy [12].

Overall, anti-ADC antibodies with various domain specificities may be induced. The value and requirement for the domain specificity characterization should be dictated by the immunogenicity risk evaluation of the compound driven by specific risk factors and requirements of the development phase of the biotherapeutic. Domain specificity characterization may be introduced in the early clinical studies in order to learn the possible differences in the impact from antibodies specific to individual domains of the ADC. Alternatively, a decision to conduct domain specificity evaluation may be made based on the totality of evidence, including immunogenicity incidence, safety and efficacy observations. A well-defined strategy for anti-ADC immunogenicity evaluation, including domain specificity assessment, is strongly suggested.

\section{Characterization of Pre-Ab responses}

Pre-Abs are a topic of considerable interest given their prevalence and potential impact on therapeutic proteins. Pre-Abs are defined herein as antibodies that are 
reactive to a biotherapeutic and exist in circulation prior to the patient receiving any dose of the biotherapeutic of interest. Detection of Pre-Ab is especially relevant in populations of subjects that have received therapy with similar products (e.g., TNF inhibitors) since cross-reactive antibodies can modify the safety and efficacy of the second (structurally similar) therapeutic. Antibodies developed upon treatment with similar product(s), although pre-exiting in nature, belong to a different category. These antibodies are usually categorized as treatment-induced antibodies and represent a different immunogenicity risk level from that of (auto) antibodies detected in treatment-naïve subjects. A Pre$\mathrm{Ab}$, regardless of its origin, could serve as a base for a memory immune response.

A higher frequency of Pre-Abs are reported in patients suffering from autoimmune conditions, for example, rheumatoid arthritis patients, suggesting that disorders with sustained inflammatory responses can have an impact in the ADA pool. Additionally, antibodies directed against bacterial, viral and yeast antigens pre-exist and can be detected in human sera regardless of their immune status. Although these antibodies are considered part of the natural antibody pool needed to protect the organism against different pathogenic agents, they could potentially cross-react with epitopes present either in the recombinant protein, for example, yeast glycans, or recognize residual impurities derived from the manufacturing process, e.g., bacterial host cell proteins, leading to unwanted antibody-mediated responses.

Autoantibodies are antibodies directed against self-proteins. Commonly detected autoantibodies are antibodies directed against cytokines involved in modulation of the immune system, for example, antiTNF antibodies in MS patients [13], but can also be antibodies implicated in autoimmune disorders (e.g., rheumatoid factor in rheumatoid arthritis patients, anti-dsDNA antibodies in SLE patients). Cross-reactive Pre-Abs are those that may be induced by exposure to one compound and can cross-react with another compound of similar structure or to an endogenous counterpart. Examples include TNF- $\alpha$ inhibitors and IFN- $\beta$. In addition to having cited examples of cross-reactivity between structurally similar therapeutics (e.g., betaseron, rebif, avonex), autoantibodies to interferons have also been reported [13,14].

Pre-Ab positive samples are those that confirm positive in the ADA assay according to the conventional multi-tiered approach [9]. It is common for ADA assays to utilize a bridging format. Therefore, in addition to ADA, it is possible for multimeric target proteins to create the bridge and produce false positive results. Since bridging assays that rely on dimeric binding have the potential to produce false-positive results due to their ability to bridge dimeric or multimeric targets [15], potential for target interference should be examined in assay development. A non-bridge assay format to confirm antibody positive status may be needed. This aspect of assay evaluation is important for both Pre-Ab and post-treatment antibody response evaluations.

When establishing the screening assay cut-point to detect ADA, it is important to differentiate between naïve patients with and without Pre-Ab. The assay cutpoint selection is challenging when high ADA signals in the pre-dose samples exist, in particular when there is a significant difference between the assay validation cut-point and the in-study (population specific) cut-point. In these cases, monitoring of treatment emergent titer changes becomes crucial.

Whether Pre-Ab prevalence should be reported in the label is a question still under active discussion. However, there is some consensus that the initial immunogenicity risk assessment should be used as guidance to determine the relevance of the detected pre-Ab. It is agreed that the prevalence of the detected pre-Ab should be described in the final clinical reports. If no post-treatment antibody titer boosting is observed, the clinical relevance of these pre-Abs will depend on the type of adverse events observed in the clinic and whether or not they can be associated to the presence of these antibodies. It is generally recommended that these pre-Abs are reported since they may help understand the nature of the immune response observed in response to the biotherapeutic.

While the same assays are usually employed to assess the presence of ADA in pre- and post-dose samples, in some instances, greater characterization may be required. For example, further characterization may be merited when an unusually high prevalence of pre- $\mathrm{Ab}$ is detected during the course of compound development or when significant differences are observed between validation and pre-dose sample analysis. Pre-validation method development should examine and mitigate for interferences mediated by circulating target and cross-reactivity events mediated by rheumatoid factor, soluble dimeric receptors, heterophilic antibodies, anticarbohydrate antibodies and antibodies to therapeutic components that are analogous to naturally occurring antigens from virus, bacteria and plants. Part of this mitigation may include greater characterization beyond the typical two-tier analysis. All supplemental characterization should be justified and supported by an immunogenicity risk assessment specific to the compound and patient population.

As with all ADAs, Pre-Abs should be understood according to the dosing time course (i.e. titer increases and other maturation events including neutralization) 
and monitored for impact on safety and efficacy [16]. Treatment-induced antibody titers should be examined in relation to Pre-Ab titers before treatment to understand 'boosting'. In general, a treatment-induced titer can be defined as an increase in titer over the pre-dose sample titer that is above the variability of the titration assay. However, the clinically relevant titer cannot be determined until clinical immunogenicity data are integrated with the PK, safety and efficacy data.

Another consideration discussed in relation to detection of Pre-Ab responses is the relevance of Pre$\mathrm{Ab}$ response in the context of new technologies and our ability to develop assays with little analytical variability. Researchers and regulators should be aware that development of assays with little analytical variability on highly sensitive technology platforms will discern slight increases in signal above the background noise of the assay as positives. Therefore, when interpreting immunogenicity data, especially in the pre-dose samples, it is imperative that attention is paid to strength of the signal and increase over time after treatment, and factor this into evaluation of clinical impact of immunogenicity. Overall, the risk analysis should provide the most appropriate rationale for the strategies to be used for Pre-Ab characterization, management and approaches to mitigate the potential impact to safety and efficacy of immunogenicity.

\section{Challenges with bioanalysis of anti-PEG antibodies \& ADA specific to PEG}

The prevalence and relevance of anti-PEG antibodies have been a topic of discussion for over a decade during presentations by various institutions at national and international meetings, various publications and the recent inclusion in the 2013 US FDA Guidance on the immunogenicity of therapeutic protein products [17]. PEGylation of therapeutic proteins is a strategy used to diminish the immunogenic potential of certain therapeutics since the presence of this polymer can mask potential antigenic epitopes present in the protein structure. Nevertheless, the extensive use of PEG in the food, cosmetic and over-the-counter treatments are believed to be the reason for the higher incidence of anti-PEG (pre-existing) antibodies among the human population [18-20].

While there still appears to be debate on the best practices for measuring anti-PEG antibodies and the clinical relevance of them, many companies are investing in a diverse set of bioanalytical approaches including the utilization of surface plasmon resonance, direct LBA, bridging LBA [21] and various technologies aimed to amplify signal, $\operatorname{IgM}$, IgG and IgE isotyping assays, commercial PCs, custom PC including humanized PC and more. The one subject that was agreed upon was that measuring anti-PEG antibody has posed technical challenges due to the repeating motif structure of PEG, the relative low affinity of many anti-PEG antibodies, the comparability of PEG structure to many detergents and the difficulty in producing a relevant PC [20]. There is evidence of clinically relevant antiPEG antibodies of the IgM and IgG isotype [22-24]. Thus, as with conventional ADA assay formats, antiPEG antibody assay formats should be able to detect a diverse set of isotypes.

Several PEGylated biologics have been approved and are used as standard-of-care therapeutics for over a decade (e.g., Pegasys and Neulasta), with significant patient benefit and with no direct evidence of adverse events or loss of efficacy attributable to the presence of anti-PEG antibodies. For these and comparable programs, it may not be valuable to launch a full-scale investigation on anti-PEG antibodies. Nevertheless, there is additional clinical evidence showing loss of efficacy and increased incidence of hypersensitivity reactions associated with detectable levels of anti-PEG antibodies of the IgM and IgG isotypes (e.g., pegasparaginase and pegloticase) [22-24], suggesting that an adequate bioanalytical strategy may be pertinent to higher risk compounds. As with all ADAs, the bioanalytical strategy should be justified by an immunogenicity risk assessment specific to a given compound and patient population.

It was agreed to follow up in a more concentrated effort to examine the pros and cons of various approaches.

\section{NAb assays}

Characterization of ADA for neutralizing potential is important to better understand the possible impact ADA can have on the biological function of the biotherapeutic and its endogenous counterpart if applicable. The timing for implementing a validated NAb assay was discussed (e.g., Phase I-III). While it is crucial to have a NAb assay by Phase III clinical development, a risk-based approach must be used in determining the stage-specific needs for any particular program. In the case of high-risk biotherapeutics, it may be important to have an NAb assay in place as early as Phase I program, whereas Phase III may be more suitable for lower risk programs. Risk is defined by the potential for incidence of ADA and the potential severity of the clinical adverse event mediated by them. High incidence alone does not drive risk. Severe consequences, however, could be a major driver in defining risk as in the case with non-redundant compounds where loss of efficacy and/or cross-neutralization of endogenous counterparts could result in severe adverse events (e.g., erythropoietin). If risk and/or mechanism 
of immune response is/are not fully understood, it is recommended to collect and bank samples at appropriate time intervals so that analysis can be conducted if needed.

The next topic discussed was when LBA might be considered in place of the more variable and labor intensive functional cell-based assays. The consensus for when LBA may be implemented in place of cellbased assays included: when the biotherapeutic mechanism was based upon a binding event as is the case with most of antagonists. This approach can also be extremely useful in bridging the gap in sensitivity of the binding ADA assay versus a cell-based NAb assay (which typically has significantly less sensitivity). In this case, a researcher is able to utilize an LBA assay format to evaluate the neutralizing potential of the binding antibodies that were detected in the more sensitive binding antibody assays. Activity assays for enzyme therapeutics were not discussed. In any case, an assay format that closely mimics the mechanism of action (MOA) in humans is recommended. Bi-specifics may require two (or more) NAb assays depending on the cumulative MOA of the biotherapeutic. Potency assays frequently provide a good basis for the cell-based NAb assay and many endpoints are suitable, including proliferation, viability, apoptosis and the use of luciferase reporter systems.

This team also discussed whether it was categorically required to have an independent assay for NAb assessment. For example, cumulative PK, PD and ADA data can be informative as to the presence of NAb and could provide as much or even more value than a standalone NAb assay which is typically less sensitive. This strategy might be relatively rare, such as in cases with a definitive absence of immune-mediated safety or clinical consequences, and should be comprehensively described in a well-developed risk assessment.

The last item for discussion concerned an NAb assay confirmatory tier. While only a few laboratories routinely do this, cases were cited where samples are run over Protein A/G columns to isolate the antibodies and remove matrix factors and are then run in NAb assay. This type of confirmation was not thought to be necessary for all programs but should be considered on a case-by-case basis.

\section{Impact of circulating drug on measuring ADA}

An apparent limitation of ADA assays that commonly use drug to capture the ADA is the potential interference of free drug circulating in the system. This interference is much more relevant to relatively high dosed therapeutics with long half-lives (e.g., mAb, Fc fusion proteins). In nonclinical safety studies and higher dose clinical situations, high concentrations of circu- lating drug will adversely impact ADA detection. It was agreed that drug tolerance should be thoroughly assessed using a surrogate PC during the ADA assay validation. While the surrogate $\mathrm{PC}$ is understood to have limitations in that it is not the best representative of the affinity and avidity of antibody found in the diverse nonclinical and clinical populations, it is presently the best source available for engineering assays that can achieve the greatest tolerance to circulating drug. More common assay strategies used to improve drug tolerance include the implementation of acid dissociation which may be followed by solid phase extraction (SPE) (e.g., SPEAD, BEAD, angiotensin-converting enzyme). A solution ELISA format also has proven to offer much higher drug tolerance even without acid treatment, a step that can sometimes compromise the ability to detect ADAs [25]. This assay format offers advantages as it can be set up using standard immunoassay platforms and is amenable to transfer across laboratories without the need for specialized equipment and training. Others include the use of biosensor systems that do not rely on two arm binding (e.g., Octet, Biacore). Additionally, immune-complex assays, such as that published by Stubenrauch et al. in 2010 [26], are innately tolerant to the presence of circulating drug and could serve as useful tools for better understanding of drug and ADA disposition. The last approach discussed was Universal Indirect Species Specific Assay (UNISA), which can be used to accelerate early preclinical immunogenicity assessment [27]. If possible, it is always recommended to collect samples for ADA assessment after therapeutic has cleared.

Several therapeutics on the market currently have post-marketing requirements centered around improved drug tolerance. It is questionable whether the increased levels of antibodies that are being detected with these improved assays are yielding clinically relevant results. These data will have to continue to be gathered and compiled to enable general conclusions to be drawn.

Finally, it was the general consensus of this team that data should be reported as 'positive' or 'negative' with the assay caveats discussed in the bioanalytical and/or nonclinical/clinical reports. As assays become more sensitive and drug tolerant, the reporting of 'inconclusive' should no longer be acceptable or standard practice as it pertains to drug tolerance. There was a general consensus that the term 'inconclusive' to report a result adds little value in this case and causes confusion in the minds of other stakeholders of the immunogenicity data (i.e. clinicians who need to interpret safety and efficacy impact, and pharmacokineticists). It should be understood that data from any analytical assay should 
be interpreted in the context of limitations of that assay as validated.

\section{PK LBA bioanalytical challenges}

The PK LBA bioanalytical challenges' discussion spanned a variety of topics and the conclusions are elaborated below. Throughout the discussions, some common themes emerged, many of which ultimately focused on critical reagents; from the practical implications of running more generic assays early in development to the challenges that arise when only suboptimal reagents are available (e.g., lack of high quality, high affinity anti-PEG antibodies).

\section{Bioanalysis of multifunctional biotherapeutics \& biotransformation of biotherapeutics}

The discussion on the bioanalysis of bispecific and multifunctional biotherapeutics focused on the correct choice of method(s) to fully understand the PK concentrationtime profiles of multi-functional domain molecules and the application of appropriate immunogenicity strategies. The general recommendation was to adopt the approaches currently employed in the bioanalysis of ADCs, which were discussed in the 2013 White Paper in bioanalysis [6]. For these types of complex molecules, multiple PK assays may be initially required in order to build an understanding of the moiety or moieties that impart clinical impact. This approach requires the generation of multiple critical reagents to enable detection of the multiple domains and potential species. While at the very earliest stages not all assays can be deployed and as reagents become available, additional assays are implemented to enable an understanding of which species are critical to monitor [28]. Once an understanding has been established, measurement of only those relevant species can be performed moving forward. It was also noted that when there are limitations on availability of critical reagents for LBA development, other bioanalytical platforms that are reagent-independent (e.g., LCMS) should be considered for PK- and ADMErelated characterization purposes.

A similar approach is recommended for the deployment of immunogenicity assays. Initially, reagent availability limitations may allow only the measurement of ADA for the whole molecule. Once appropriate reagents become available, then the specificity of the ADA response can be tested by performing competition assays to understand the domain(s) to which immunogenicity is directed. Later in development, as for PK assays, the focus can be on clinically relevant measurements.

Moreover, the topic of biotransformation of biotherapeutics and the employment of orthogonal techniques to understand the complexities of biotransformation were discussed. In this arena it was also agreed that the stage of development dictates which analytes are important to track and the primary goal is to understand the moieties that are active and relevant to safety and efficacy. For example, if metabolites are the active species, then they will need to be measured throughout drug development.

\section{Challenges with bioanalysis of PEGylated proteins} by LBA

Bioanalysis of PEGylated proteins by LBA highlighted additional challenges associated with complex biotherapeutics. Details and case studies on PEG-specific analyses were reviewed in depth during the workshop. Considerations for PK assay development and validation were similar to those for other complex molecules, with multiple assays employed during the discovery/ early development phases as characterization tools to understand relative levels of intact molecule, total protein, free PEG and so on. Once it has been determined that free PEG is not an issue, for example, then there is no need to continue to routinely monitor PEG moving forward, although periodic verifications can certainly be performed if desired.

Critical reagents pose a particular challenge in this arena as PEG reagents are notoriously suboptimal, which limits their utility. No single anti-PEG antibody works well for all PEGs, although some may work well for a subset of applications. There is therefore a need to generate multiple anti-PEG antibodies with no guarantee that they will be adequate to achieve the desired assay sensitivity. Therefore, once characterization assays have been completed, a single assay that measures only the total protein is preferred when moving forward into the regulated environment. If deemed prudent, then verification of PEG integrity can be performed if desired.

There were no specific recommendations for preferred assay formats in the analysis of PEGylated proteins. Instead, it was highlighted that the assays employed during characterization may span multiple formats/orthogonal methods.

Lastly, it was discussed whether free PEG is considered as source of potential interference in the PK assay. The agreement was that to date, this has not been considered an issue, generally because free PEG levels relative to intact protein levels are believed to be extremely low. It was also noted that if significant levels of free PEG were a concern, this would have been identified when characterization experiments were performed during the discovery phase.

\section{Transition from early to later PK assays}

Discussions on complexities associated with transition from early to later stage assays again began with 
an acknowledgment that changes in assay formats are inevitable as drug development progresses, particularly for complex modalities. Assays in support of earlier phases will typically be restricted by the availability of critical reagents. For example, preclinical PK studies may be supported using a generic PK assay, or in the case of an ADC, the total molecule assay may be the only available assay. As development progresses and reagents are generated, more specific or multiple assays may be employed. For complex biotherapeutics, a series of assay changes may occur, starting with fewer assays based upon available reagents, then transitioning to multiple assays while building an understanding of the most relevant biotherapeutic species, and then finally scaling back to fewer assays again once the most relevant species to monitor throughout later stages of development have been identified. It was agreed that it is likely that changes in PK profiles will occur as new, more specific critical reagents become available with the evolution of methods. In fact, this should be expected when the final assay format moving forward is focused only on measuring the specific species of interest rather than multiple species. The consensus view was that it is not necessary to have the same result from different PK assays, but rather that there should be a scientific understanding of the species that the assays are measuring so that results can be interpreted appropriately. It was noted, however, that when feasible, keeping the dose-ranging assay and the first-in-human assay as similar as possible will simplify interpretation.

\section{Overcoming interferences in PK LBA}

A comprehensive overview and specific case studies on overcoming assay interferences were discussed, which mainly focused on predicting interferences, evaluating them during assay development and validation (both pre- and in-study), and strategies to mitigate interferences. Certain interferences can be predicted based on the assay format and available critical reagents, with examples being interferences from soluble target or ADA. These predicted interferences therefore play into reagent and assay format selection. The recommendation was to be aware of what may potentially be encountered and evaluate accordingly. However, since these pre-study evaluations will be imperfect, close attention should be paid to data from 'real' samples and any unusual or unexpected results should be investigated accordingly. For example, evaluating potential interference of soluble ligand with recombinant soluble ligand may not accurately reflect the effects of endogenous ligand, especially if the therapeutic has been raised against the recombinant ligand. When unpredicted interferences arise from matrices, different assay platforms may need to be evaluated to mitigate the nonspecific binding. For example, switching from a conventional plate-based format to a microfluidic system was discussed during the workshop.

Robust discussion occurred and no clear consensus was achieved regarding whether free (measuring unbound and partially bound, or active, therapeutic) or total PK assays [29] are preferred within the scope of interference-related issues; especially in the context of preclinical studies. In the discovery and preclinical spaces, some companies are primarily using free assays while others are running total assays. Interestingly, some companies have switched their practices in recent years, indicating that this is an evolving area. However, there was an agreement that availability of reagents frequently drives the decision on assay format selection as do the opinions of the PK scientists, PK/PD modelers and toxicologists. It was also agreed that there are pros and cons associated with both total and free assays. The group also highlighted the need to be cognizant of the perturbations in equilibrium caused by dilution of samples. For example, high concentration samples requiring large dilutions may drive bound drug to the unbound state and measurements derived from a free assay format may therefore include a proportion of drug that was in a bound state in vivo.

The pros and cons of employing LCMS versus LBA and whether interference issues with LBA justify switching to LCMS methods were also discussed. It was agreed that there may be multiple approaches that could be applied to mitigate interference issues, including LCMS. Whether it is justified to switch to LCMS would need to be determined on a case-by-case basis. In the panelists' personal experience, LCMS has more frequently been employed as a tool to facilitate understanding of the interferences. With that understanding in hand, then the appropriate assay format is chosen and implemented. It was agreed that it is desirable to deploy only one assay, rather than multiple assays, especially in later stages of clinical development.

\section{PK assay \& immunogenicity}

During the discussion on interferences, particular attention was given to $\mathrm{ADA}$ interference in PK assays $[30,31]$. Dissociation techniques, such as acid treatment, are frequently employed in ADA assays to increase the drug tolerance of the assay. Such techniques can also be used to mitigate ADA interference in PK assays. While it is theoretically possible to achieve complete dissociation, it was agreed that maintenance of this dissociation is questionable. In addition, acid treatment may inactivate the therapeutic and result in reported drug levels being lower than they actually are. For example, acid treatment may fully dissociate complexes, but some re-association will occur upon neutralization. 
Therefore, the recommendation was to be aware of the limitations of both the dissociation method and the assay. It was also noted that, practically speaking, differences in measurement of samples that are nearly completely dissociated versus truly completely dissociated may be negligible if the differences are within the assay's bioanalytical variability.

Given that PK assays often employ anti-idiotypic antibodies or soluble target as capture reagents, it is widely acknowledged that ADAs have the potential to interfere in the assay [31]. In addition, ADA to other epitopes of the therapeutic can also interfere with binding of the detection reagent or cause steric hindrance. However, routine assessment of ADA interference in PK assays is not recommended. Instead, it is important to understand the PK assay and what it is capable of measuring and to also consider data from other relevant assessments (e.g., PD and efficacy). Although there may be times where there is good scientific rationale to evaluate for ADA interference, there are caveats with these evaluations since the PCs that are typically employed are imperfect surrogates for genuine ADA. The assessments are therefore not expected to provide meaningful information with respect to in vivo responses. Additionally, there is no need to routinely employ strategies to mitigate ADA interference, although there should be an understanding of how ADA may impact PK results.

\section{Follow-up on LBA part of the 2013 draft FDA Guidance on BMV}

The appropriate calculation of the nominal concentration of protein therapeutics was one of the points discussed in relation to the draft FDA Guidance. It was agreed that protein concentrations should be derived from mass-based methods only. It was indicated that this recommendation is in alignment with best practices at their companies. While protein purity and activity are typically assessed as part of lot release processes, no adjustment in nominal protein concentration should be made.

The question on whether it is useful to include additional QC samples when study sample concentrations are clustered in a narrow range of the standard curve was debated. At the 2013 AAPS Workshop (Crystal City V), it was agreed that ideally the bulk of sample results will be bracketed by QC samples with an acknowledgement that, generally speaking, LBA QC samples tend to already be 'close' to each other. Additionally, when sample dilutions are chosen to target sample results in the middle of the curve then it is expected that data will cluster there. It was further clarified that clustering of sample concentrations in the middle of the calibration curve is actually desirable.
Furthermore it was agreed that there is no scientific rationale for inclusion of additional QC samples in $\mathrm{PK}$ LBAs as it is not possible for the entire standard curve and all the QC samples to pass criteria and yet have a subsection of the curve be inaccurate. It was agreed that adding more QC samples would neither add value nor alter the assay results. However, special consideration was given for cases where the bulk of the data fall between Low QC and the LLOQ. This would occur rarely for LBA PK assays and only if all samples from a given study were tested at minimum required dilution only and the results were unexpectedly low. It was agreed that in this case, the suitability of the assay's range would be in question. In contrast, exploratory biomarker assays may commonly have sensitivity challenges that will result in data clustered at the lower end of the standard curve. In these cases, the level of decision making supported by the data would be impacted. Improvements in the assay would be required if the biomarker continued to be pursued into later stages of drug development and was intended to inform clinical decisions.

As new technologies and automation capabilities become available, and as assays evolve and better reagents become available, the question arises as to whether the industry and regulators are ready for the implementation of singlet analysis for LBAs. This topic was also discussed at the 2013 AAPS Workshop (Crystal City V) and the recommendations herein are consistent with the expressed industry position at that conference. Essentially the decision to employ singlet analysis should be approached in a risk-based manner and implementation left to the judgment of the bioanalytical team. There was consensus that singlet analysis should be acceptable if scientifically justified (e.g., based on the performance of the assay used). It was also noted that the generation (and presentation) of data to support singlet analysis will help to gain traction as an acceptable practice.

The overarching conclusion on LBA bioanalytical challenges was that good science must drive decision making whereby strong scientific rationale and relevant data sets should be generated to justify the decisions made. In effect, good science wins the day. Over the course of discussions, the recommendation of "Do what you have to do to understand what you need to understand to ensure you do the right thing" was developed. There is some merit in this superficially simplistic view. To illustrate:

- Do what you have to do (e.g., run multiple assays/ formats/orthogonal methods);

- To understand what you need to understand (e.g., clinical relevance - efficacy/safety); 
- To ensure you do the right thing (e.g., measure the relevant analytes);

- At the right time (needs in the discovery phase differ from needs in late stage drug development).

Another theme that arose through multiple discussions over the course of the workshop was "don't do something just because you can". The recurring recommendation was to be scientifically driven. When anomalous data arise, bioanalytical scientists need to ensure that they not only understand the assays and their limitations but also the biology of the pathway the therapeutic molecule is targeting. Although any issues that may be attributed to assay limitations will need to be mitigated, consideration should be given also that the assay may not need to be fixed. It may in fact be imparting important information related to the biology.

\section{Conclusion}

Below is a summary of the recommendations made during the 8th WRIB.

\section{Large molecules by LBA recommendations}

\section{Immunogenicity}

- ADCs are only one of many classes of multiple functional domain biotherapeutics. As with other compounds with complex multi-domain structures and potentially multistep modes of action, ADCs require a series of immunogenicity tests to fully understand the immunogenic profile. The current thinking is that specificity testing should be conducted for each domain with a functional endpoint, for example, the antibody and payload. Further testing may be merited based upon a customized risk-assessment.

- Pre-Abs are antibodies that bind to therapeutic before the patient has undergone therapy. Prevalence and impact should be examined during the course of immunogenicity testing. In some circumstances, additional characterizations may be required (e.g., antibody verification, isotyping, epitope reactivity and/or functional domain reactivity).

- The measurement of anti-PEG antibodies has been addressed as being technically challenging due to the repeating motif structure of PEG, the relative low affinity of many anti-PEG antibodies, the comparability of PEG structure to many detergents and the difficulty in producing robust and relevant PCs. Although the bioanalytical methods remain imperfect and non-harmonized across the industry, enough case study data exist where anti-PEG antibodies have been shown to have adverse effects on therapeutic efficacy such that routine assessment is needed for all PEGylated biotherapeutics. The bioanalytical community should continue to publish and share data on this topic since PEG is a common structure used by many companies and industries to yield various advantages.

- NAb assays are required to support Phase III clinical testing and, depending on the customized risk assessment for a given compound, may be merited earlier. A functional bioassay is frequently preferred but there may be several situations where a binding assay may be more suitable, for example, when the MOA is based upon binding or there is large difference in the sensitivity of the binding ADA assay versus a cell-based NAb assay. Additionally, it can be quite useful to have PD markers which also can indicate the presence of NAbs and to establish therapeutic activity in vivo.

- Drug tolerance is a frequently encountered challenge amongst relatively high dosed therapeutics with a long half-life. However, a great deal of progress has been made within the bioanalytical community in the last decade concerning the technical hurdles, possibly due to the number of post-marketing requirements from health authorities, thus making drug tolerance a routine objective for all clinical testing.

\section{PK LBA bioanalytical challenges}

- For the bioanalysis of bi-specific and multifunctional biotherapeutics, multiple PK assays using several critical reagents may be required at an early stage in order to build an understanding of the moieties that impart clinical impact. Once an understanding has been established, measurement of only those relevant species can be performed moving forward. The stage of development dictates which analytes are important to monitor and the primary goal is to understand the moieties that are active and relevant to safety and efficacy.

- The assays employed during characterization of PEGylated proteins by LBA may span multiple formats/orthogonal methods. While still in the discovery phase, multiple assays should be employed as characterization tools to understand relative levels of the different free and bound forms present. Because of the difficulty in obtaining anti-PEG antibodies that allow for desired assay sensitivity, once characterization assays have been completed, 
a single assay that measures only the total protein is preferred when moving forward into the regulated environment.

- Changes in assay formats are inevitable when moving from early to later stage assays. Assays in support of earlier phases will typically be restricted by available critical reagents. It is likely that changes in PK profiles will occur as new, more specific critical reagents become available with the evolution of methods. It is not necessary to have the same result from different PK assays, but rather that there should be a scientific understanding of the species that the assays are measuring so that results can be interpreted appropriately.

- Certain interferences in LBA can be predicted based on the assay format and available critical reagents, therefore one should be aware of what may potentially be encountered and evaluate/ investigate accordingly. There are pros and cons associated with both total and free assays, and there may be multiple approaches that could be applied to mitigate interference issues, including the use of LCMS. It is desirable to deploy only one assay, rather than multiple assays, especially in later stages of clinical development.

- Dissociation techniques, such as acid treatment, are frequently employed in ADA assays to increase the drug tolerance of the assay. As the maintenance of this dissociation is questionable, the limitations of both the dissociation method and the assay should be considered. Moreover, acid treatment may inactivate the therapeutic and result in reported drug levels lower than they actually are. Routine assessment of ADA interference in PK assays is not recommended; however, it is important to understand the PK assay and to also consider data from other relevant assessments (e.g., PD and efficacy). Additionally, there is no need to routinely employ strategies to mitigate ADA interference, although there should be an understanding of how ADA may impact PK results.

- In relation to the LBA section of the $2013 \mathrm{draft}$ FDA Guidance on BMV, while protein purity and activity are typically assessed as part of lot release processes, protein concentrations should be derived from mass-based methods only. Regarding the inclusion of additional QC samples in PK LBAs, this is not considered to add any scientific value nor alter the assay results. Finally, the decision to employ singlet analysis for LBAs should be approached in a risk-based manner. Singlet analysis should be acceptable if scientifically justified (e.g., based on the performance of the assay used).

\section{Acknowledgements}

The authors would like to acknowledge the US FDA, Europe EMA, Health Canada, France ANSM, The Netherlands MEB, Austria AGES, Germany BfArM, Japan MHWL and UK MHRA for supporting this workshop. L Stevenson (Biogen Idec), L Amaravadi (Biogen Idec), H Myler (Bristol-Myers Squibb), D Dufield (Pfizer), L Salazar-Fontana (FDA), H Neubert (Pfizer), L Kirkovsky (Pfizer), F Garofolo (Algorithme Pharma), I Dumont (Algorithme Pharma), B Gorovits (Pfizer), E Fluhler (Pfizer), R Hayes (MPI Research), K Xu (Genentech), M Arnold (Bristol-Myers Squibb), $S$ Bansal (Roche), T Verhaeghe (Janssen), A Bergeron (Algorithme Pharma), O Laterza (Merck), SC Alley (Seattle Genetics), M Szapacs (GlaxoSmithKline), A Wilson (AstraZeneca), S Kaur (Genentech) and A Joyce (Pfizer), for their major contribution in this White Paper. E Fluhler (Pfizer), O Le Blaye (France ANSM), D Dufield (Pfizer), L Amaravadi (Biogen Idec), L Stevenson (Biogen Idec) and F Garofolo (Algorithme Pharma) for chairing the workshop. E Fluhler (Pfizer), D Dufield (Pfizer), L Amaravadi (Biogen Idec) and $L$ Stevenson (Biogen Idec) for chairing the White Paper discussions. All the workshop attendees and members of the bioanalytical community who have sent comments and suggestions to complete this White Paper. W Garofolo, L Lu, X Wang, E Petrova, M Losauro and B Felcyn for the logistic assistance of the event. Future Science Group as a trusted partner.

\section{Financial \& competing interests disclosure}

The authors have no relevant affiliations or financial involvement with any organization or entity with a financial interest in or financial conflict with the subject matter or materials discussed in the manuscript. This includes employment, consultancies, honoraria, stock ownership or options, expert testimony, grants or patents received or pending, or royalties.

No writing assistance was utilized in the production of this manuscript.

\section{Author Afililations}

${ }^{\ddagger}$ Co-author for topics 2 and 3 of the immunogenicity section

${ }^{\S} \mathrm{Co}$-author for the immunogenicity section

'Biogen Idec Inc., Cambridge, MA, USA

${ }^{2}$ Bristol-Myers Squibb, Princeton, NJ, USA

${ }^{3}$ US FDA, Silver Spring, MD, USA

${ }^{4}$ Pfizer, Andover, MA, USA

\author{
${ }^{5}$ Algorithme Pharma, Inc., 575 Armand-Frappier Blvd., Laval \\ (Montreal), QC H7V 4B3, Canada \\ ${ }^{6}$ Seattle Genetics Inc., Bothell, WA, USA \\ ${ }^{7}$ Amgen Inc., Thousand Oaks, CA, USA
}


${ }^{8}$ Roche Innovation Center, New York, NY, USA

9inVentiv Health Clinical, Montreal, QC, Canada

${ }^{10}$ Merck, Kenilworth, NJ, USA

${ }^{11}$ Tandem Labs, West Trenton, NJ, USA

${ }^{12}$ Pfizer, Pearl River, NY, USA

${ }^{13}$ Pfizer, Groton, CT, USA

${ }^{14}$ Intertek, San Diego, CA, USA - Presently working at BioAgilytix Labs

${ }^{15}$ Allergan Inc., Irvine, CA, USA

${ }^{16} \mathrm{MPI}$ Research, Mattawan, MI, USA

${ }^{17}$ Merck, Oss, The Netherlands - Presently working at Quintiles

${ }^{18}$ Japan MHLW-NIHS, Tokyo, Japan

\section{References}

1 Savoie N, Booth BP, Bradley T et al. 2008 White Paper: the 2nd calibration and validation group workshop on recent issues in good laboratory practice bioanalysis. Bioanalysis 1(1), 19-30 (2009).

2 Savoie N, Garofolo F, van Amsterdam P et al. 2009 White Paper on recent issues in regulated bioanalysis from the $3 \mathrm{rd}$ calibration and validation group workshop. Bioanalysis 2(1), 53-68 (2010).

3 Savoie N, Garofolo F, van Amsterdam P et al. 2010 White Paper on recent issues in regulated bioanalysis and global harmonization of bioanalytical guidance. Bioanalysis 2(12), 1945-1960 (2010).

4 Garofolo F, Rocci M, Dumont I et al. 2011 White paper on recent issues in bioanalysis and regulatory findings from audits and inspections. Bioanalysis 3(18), 2081-2096 (2011).

5 DeSilva B, Garofolo F, Rocci M et al. 2012 White Paper on recent issues in bioanalysis and alignment of multiple guidelines. Bioanalysis 4(18), 2213-2226 (2012).

6 Stevenson L, Rocci M, Garofolo F et al. 2013 White Paper on recent issues in bioanalysis: 'hybrid' - the best of LBA \& LC/MS. Bioanalysis 5(23), 2903-2918 (2013).

7 Gorovits B, Wakshull E, Pillutla R et al. Recommendations for the characterization of immunogenicity response to multiple domain biotherapeutics. J. Immunol. Methods 408, 1-12 (2014).

8 Mitchison NA. The carrier effect in the secondary response to hapten-protein conjugates. II. Cellular cooperation. Eur. J. Immunol. 1(1), 18-27 (1971).

9 Shankar G, Devanarayan V, Amaravadi L et al. Recommendations for the validation of immunoassays used for detection of host antibodies against biotechnology products. J. Pharm. Biomed. Anal. 48, 1267-1281 (2008).

10 Hoofring SA, Lopez R, Hock MB et al. Immunogenicity testing strategy and bioanalytical assays for antibody-drug conjugates. Bioanalysis 5(9), 1041-1055 (2013).

11 Carrasco-Triguero M, Yi JH, Dere R et al. Immunogenicity assays for antibody-drug conjugates: case study with adotrastuzumab emtansine. Bioanalysis 5(9), 1007-1023 (2013).

12 Girish S, Gupta M, Wang B et al. Clinical pharmacology of trastuzumab emtansine (T-DM1): an antibody-drug conjugate in development for the treatment of HER2positive cancer. Cancer Chemother. Pharmacol. 69, 12291240 (2012).

13 Watanabe M, Uchida K, Nakagaki K et al. High avidity cytokine autoantibodies in health and disease: pathogenesis
${ }^{19} \mathrm{Genentech}$, South San Francisco, CA, USA

${ }^{20}$ Merck, Whitehouse Station, NJ, USA

${ }^{21}$ inVentiv Health Clinical, Quebec City, QC, Canada

${ }^{22}$ Roche Innovation Center, Basel, Switzerland

${ }^{23}$ Quintiles, Ithaca, NY, USA

${ }^{24}$ UCB Pharma, Braine-l'Alleud, Belgium

${ }^{25}$ Regeneron Pharmaceuticals, Tarrytown, NY, USA

${ }^{26}$ UK MHRA, London, UK

${ }^{27}$ AstraZeneca, Macclesfield, UK

${ }^{28}$ Merck Research Laboratories, West Point, PA, USA

${ }^{29}$ Janssen Research \& Development, Spring House, PA, USA

and mechanisms. Cytokine Growth Factor Rev. 21, 263-273 (2010).

14 Polman CH, Bertolotto A, Deisenhammer F et al. Recommendations for clinical use of data on neutralising antibodies to interferon-beta therapy in multiple sclerosis. Lancet Neurol. 7, 740-750 (2010).

15 Bautista AC, Wullner D, Moxness $M$ et al. Impact of matrix-associated soluble factors on the specificity of the immunogenicity assessment. Bioanalysis 2(4), 721-731 (2010).

16 Xue L, Rup B. Evaluation of pre-existing antibody presence as a risk factor for posttreatment anti-drug antibody induction: analysis of human clinical study data for multiple biotherapeutics. AAPS J. 15(3), 893-896 (2013).

17 US Department of Health and Human Services, US FDA, Center for Drug Evaluation and Research, Center for Biologics Evaluation and Research. Draft guidance for industry, immunogenicity assessment for therapeutic protein products. Rockville, MD, USA (2013). www.fda.gov/downloads/drugs/ guidancecomplianceregulatoryinformation/guidances/ ucm338856.pdf.

18 Garay RP, El-Gewely R, Armstrong JK et al. Antibodies against polyethylene glycol in healthy subjects and in patients treated with PEG-conjugated agents. Expert Opin. Drug Deliv. J. 9(11), 1319-1323 (2012).

19 Garay RP, Labaune JP. Immunogenicity of polyethylene glycol (PEG). Open Conf. Proc. J. 2, 104-107 (2011).

20 Schellekens H, Hennink WE, Brinks V. The immunogenicity of polyethylene glycol: facts and fiction. Pharm. Res. 30(7), 1729-1734 (2013).

21 Liu Y, Reidler H, Pan J et al. A double antigen bridging immunogenicity ELISA for the detection of antibodies to polyethylene glycol polymers. J. Pharmacol. Toxicol. Methods 64(3), 238-245 (2011).

22 Armstrong JK, Hempel G, Koling S et al. Antibody against poly(ethylene glycol) adversely affects PEG-asparaginase therapy in acute lymphoblastic leukemia patients. Cancer 110(1), 103-111 (2007).

23 Ganson NJ, Kelly SJ, Scarlett E et al. Control of hyperuricemia in subjects with refractory gout, and induction of antibody against poly(ethylene glycol) (PEG), in a phase I trial of subcutaneous PEGylated urate oxidase. Arthritis Res. Ther. 8(1), R12 (2006).

24 Hershfield MS, Ganson NJ, Kelly SJ et al. Induced and preexisting anti-polyethylene glycol antibody in a trial of every 3-week dosing of pegloticase for refractory gout, including 
in organ transplant recipients. Arthritis Res. Ther. 16(2), R63 (2014).

25 Mikulskis D, Yeung M, Subramanyam L, Amaravadi, . Solution ELISA as a platform of choice for development of robust, drug tolerant Immunogenicity assays in support of drug development. J. Immunol. Methods 365, 38-49 (2011).

26 Stubenrauch K, Wessels U, Essig U et al. Evaluation of a generic immunoassay with drug tolerance to detect immune complexes in serum samples from cynomolgus monkeys after administration of human antibodies. J. Pharm. Biomed. Anal. 52, 249-254 (2010).

27 Bautista AC, Salimi-Moosavi H, Jawa V. Universal immunoassay applied during early development of large molecules to understand impact of immunogenicity on biotherapeutic exposure. AAPS J. 14(4), 843-849 (2012).

28 Weidle UH, Tiefenthaler G, Weiss EH, Georges G, BrinkmannU. The intriguing options of multispecific antibody formats for treatment of cancer. Cancer Genomics Proteomics 10(1), 1-18 (2013).

29 Lee J W, Kelley M, King L E et al. Bioanalytical approaches to quantify 'total' and 'free' therapeutic antibodies and their targets: technical challenges and PK/PD applications over the course of drug development. AAPS J. 13(1), 99-110 (2011).

30 Sailstad JM, Amaravadi L, Clements-Egan A et al. A White Paper - consensus and recommendations of a global harmonization team on assessing the impact of immunogenicity on pharmacokinetic measurements. AAPS J. 3, 488-498 (2014).

31 Kelley M, Ahene AB, Gorovits B et al. Theoretical considerations and practical approaches to address the effect of anti-drug antibody (ADA) on quantification of biotherapeutics in circulation. AAPS J. 3, 646-658 (2013). 